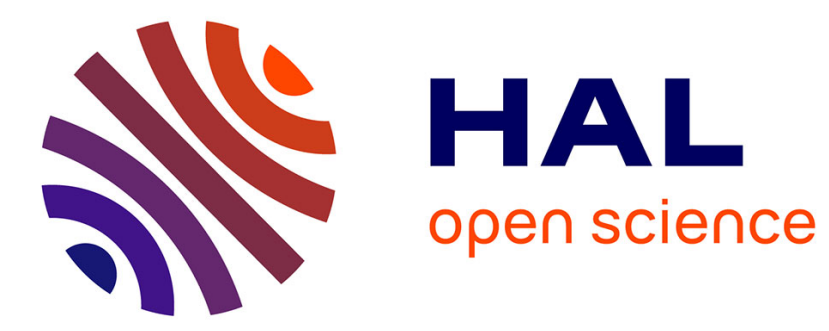

\title{
Les ruses de la raison budgétaire
}

\author{
Constantin Brissaud, Pierre-André Juven
}

\section{To cite this version:}

Constantin Brissaud, Pierre-André Juven. Les ruses de la raison budgétaire. Actes de la Recherche en Sciences Sociales, 2020. halshs-03081810

\section{HAL Id: halshs-03081810 https://shs.hal.science/halshs-03081810}

Submitted on 18 Dec 2020

HAL is a multi-disciplinary open access archive for the deposit and dissemination of scientific research documents, whether they are published or not. The documents may come from teaching and research institutions in France or abroad, or from public or private research centers.
L'archive ouverte pluridisciplinaire HAL, est destinée au dépôt et à la diffusion de documents scientifiques de niveau recherche, publiés ou non, émanant des établissements d'enseignement et de recherche français ou étrangers, des laboratoires publics ou privés. 


\title{
LES RUSES DE LA RAISON BUDGÉTAIRE
}

\section{L'expertise de l'OCDE sur le prix du médicament}

\author{
Constantin Brissaud, Pierre-André Juven
}

Le Seuil | «Actes de la recherche en sciences sociales 》

$2020 / 4 N^{\circ} 234 \mid$ pages 34 à 49

ISSN 0335-5322

ISBN 9782021442526

Article disponible en ligne à l'adresse :

https://www.cairn.info/revue-actes-de-la-recherche-en-sciencessociales-2020-4-page-34.htm

Distribution électronique Cairn.info pour Le Seuil.

(C) Le Seuil. Tous droits réservés pour tous pays.

La reproduction ou représentation de cet article, notamment par photocopie, n'est autorisée que dans les limites des conditions générales d'utilisation du site ou, le cas échéant, des conditions générales de la licence souscrite par votre établissement. Toute autre reproduction ou représentation, en tout ou partie, sous quelque forme et de quelque manière que ce soit, est interdite sauf accord préalable et écrit de l'éditeur, en dehors des cas prévus par la législation en vigueur en France. Il est précisé que son stockage dans une base de données est également interdit. 


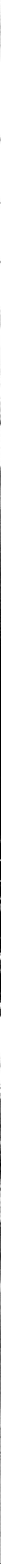

GRAFFITI PRÉSENT rue Alfred Dehodencq, attenante aux bâtiments de l'OCDE, à Paris 16e . 


\title{
Constantin Brissaud et Pierre-André Juven
}

\section{Les ruses de la raison budgétaire}

\author{
L'expertise de l'OCDE sur le prix du médicament
}

Au cœur de multiples controverses, les médicaments innovants sont pour la grande majorité développés par des laboratoires pharmaceutiques situés dans les pays de l'OCDE, mais leurs prix sont négociés au cas par cas, au niveau des États. L'industrie pharmaceutique représente de nombreux emplois, et d'importantes rentrées fiscales, notamment aux États-Unis. Dès lors, la politique du médicament est simultanément une politique de santé publique et une politique industrielle ${ }^{1}$. Si les pays de l’OCDE n'ont, jusqu'à récemment, jamais connu de réelles difficultés à financer les innovations thérapeutiques - cantonnant le problème du coût des médicaments aux pays des Suds -, la mise sur le marché fin 2013 d'un traitement contre l'hépatite C, le Sovaldi, à un prix jugé extrêmement élevé par de nombreux acteurs, a conduit plusieurs pays industrialisés à constituer le prix des traitements innovants en problème public ${ }^{2}$. Les solutions politiques à apporter à ce problème sont un enjeu de luttes que cet article entend exposer en analysant l'écriture d'un rapport par une institution internationale centrale dans l'élaboration des lieux communs guidant les réformes des systèmes de santé des pays industrialisés : l'Organisation de coopération et de développement économiques (OCDE).

L'objectif de l'OCDE est de formuler des propositions pour résoudre un problème présenté sous la forme d'un triptyque : comment assurer le retour sur investissement des laboratoires pharmaceutiques et stimuler « l'innovation » pour trouver de nouveaux traitements ; comment rendre accessibles les médicaments arrivant sur le marché ; comment concilier ces deux objectifs avec la nécessité de limiter les dépenses de santé, dont le rappel, au début de chaque rapport consacré à la santé, a, depuis le milieu des années 1980, permis à l'OCDE de s'imposer comme une organisation internationale légitime sur ce thème ${ }^{3}$. Débordant son rôle traditionnel d'établissement de statistiques comparatives des dépenses, la division santé de l'OCDE produit, à partir des années 2000, des recommandations en matière de régulation du prix du médicament. Le sujet s'impose dans l'agenda de la division à mesure que l'élévation des prix met en danger les systèmes sociaux des États dans les années 2010 : le péril que font subir aux dépenses publiques les coûts extrêmement élevés des « médicaments innovants » nécessite en effet, pour tenir le credo de la limitation des dépenses publiques, des dispositifs renouvelés.

À ce titre, la division santé de l'OCDE publie en janvier 2017 un rapport ${ }^{4}$ incluant une réflexion sur les enjeux relatifs aux prix des innovations thérapeutiques. Ce rapport ne doit être qu'une étape intermédiaire vers un rapport « final » devant initialement être publié fin 2017 et qui est finalement publié en
1. Boris Hauray, L'Europe du médicament. Politique - Expertise - Intérêts privés, Paris, Presses de Sciences Po, 2006 ; Étienne Nouguez, Des médicaments à tout prix. Sociologie des génériques en France, Paris, Presses de Sciences Po, 2017.
2. Fanny Chabrol, Pierre-Marie David et Gaëlle Krikorian, "Rationing hepatitis $\mathrm{C}$ treatment in the context of austerity policies in France and Cameroon: a transnational perspective on the pharmaceuticalization of healthcare systems", Social Science \&
Medicine, 187, 2017, p. 243-250.

3. Constantin Brissaud, "La production internationale d'un sens commun réformateur. Concurrences expertes et argument statistique de la "crise" des dépenses de santé à l'OCDE (1972-2018) ", thèse de doctorat en science politique, Strasbourg, Université de Strasbourg, 2019.

4. OCDE, New Health Technologies. Managing Access, Value and Sustainability, Paris, OECD, 2017. 
novembre $2018^{5}$. Par souci de clarté et même si les rapports ne s'intitulent pas ainsi, nous mentionnerons ces deux rapports sous les titres de « Rapport 1 » pour celui de janvier 2017 et de « Rapport 2 » pour celui de novembre $2018^{6}$. Notre étude, qui porte sur cette période, met au jour les négociations qui ont débouché sur le rapport final. L'enquête porte sur l'opposition, au sein d'une institution internationale, de camps défendant des options politiques antagonistes quant à la régulation des prix des médicaments dits innovants. Elle analyse le rôle des pays membres, de l'industrie pharmaceutique, des syndicats, et des ONG dans le processus de production d'un savoir international sur un sujet politiquement clivant, afin de réinterroger la question de l'influence.

Deux lectures du problème s'opposent au sein de l'OCDE. L'une est portée par les représentants des laboratoires pharmaceutiques protégés par le régime des brevets, par des pays membres comme les États-Unis ou le Japon, et par une partie de l'OCDE, notamment la division des affaires économiques (ECO). Elle considère que le niveau des prix n'est pas un problème mais bien plutôt une solution qui incite à l'innovation et à la recherche de nouveaux traitements. Selon cette lecture, les prix assurent également le dynamisme d'un secteur industriel pourvoyeur de croissance, d'emplois et de recettes fiscales. L'autre lecture est portée par la division santé de l'OCDE, mais aussi par des ONG, les organisations syndicales et certains pays membres comme l'Autriche. Ils jugent le prix de certains médicaments excessif au vu de leur valeur thérapeutique. Selon eux, les largesses passées en matière de régulation doivent être sérieusement revues et il convient désormais de contraindre les laboratoires pharmaceutiques à contenir les prix.

Notre enquête suit un double objectif : premièrement, montrer comment une organisation internationale historiquement favorable à la liberté du marché se trouve prise en tenaille entre profitabilité du secteur marchand et rigueur des dépenses publiques ; deuxièmement, saisir les effets produits par des acteurs marchands et militants sur la production du savoir de cette organisation, sa doctrine politique et économique. L'article dévoile alors les asymétries de ressources, notamment en termes d'effectifs et de moyens des différents protagonistes. Au vu du contenu modéré du rapport publié en 2018 (le « Rapport 2 »), il s'agit donc de mettre en lumière les processus de production d'une position officielle, d'un « compromis » dans le « lieu neutre » que constitue l'OCDE Ce faisant, nous adoptons sur les « lieux neutres » une approche processuelle, en montrant comment les positions qui y sont prises sont, sous l'effet des rapports de force politiques et économiques qui sous-tendent les recommandations délivrés ${ }^{8}$, progressivement dépouillées de toute tonalité critique vis-à-vis des acteurs les plus puissants au sein de l'organisation et vis-à-vis des acteurs industriels.

La première partie de l'article porte sur la production de l'expertise de l'OCDE sur le prix des médicaments depuis les années 1990. Nous montrons que cette expertise doit, pour se rendre crédible, composer avec des controverses diplomatiques, des concurrences, internes à l'Organisation entre départements, et entre l'OCDE et les autres organisations internationales (OI). Nous documentons la difficulté structurelle dans laquelle se trouve plongée la division santé à produire un savoir critique. La deuxième partie s'intéresse plus particulièrement aux années 2010 et au retour sur le devant de la scène de la question des prix des innovations thérapeutiques, notamment au moment de la crise du Sovaldi, traitement contre l'hépatite C dont le prix particulièrement élevé fait scandale ${ }^{9}$. Dans cette partie nous montrons comment des employée•·s de la division santé cherchent à faire évoluer sa doctrine en tentant de formuler des recommandations plus contraignantes pour l'industrie pharmaceutique. La troisième partie décrit le processus d'écriture du rapport de l'OCDE sur les médicaments paru fin $2018^{10}$ (le « Rapport 2 ») et montre comment les contraintes qui s'exercent sur ses rédacteurs et rédactrices diffèrent la publication du rapport de près d'un an et le vouent à ne contenir que des solutions convenant aux parties les plus influentes à l'OCDE : les industriels du médicament et les pays membres les plus riches, notamment les États-Unis.

Parce que notre enquête articule dans une première partie une approche socio-historique de la place de la santé à l'OCDE et dans les deuxième et troisième parties une analyse sociologique des controverses dans la production contemporaine d'une expertise, notre méthodologie consiste en deux modes de collecte de matériaux essentiels : l'analyse d'archives et de littérature grise d'une part, des observations ethnographiques d'autre part. Les archives proviennent
5. OCDE, Pharmaceutical Innovation and domaine du médicament ou plus largement Access to Medicines, Paris, OECD Health de l'industrie pharmaceutique. Policies Studies, 2018.

6. Voir également l'encadré chronologique [voir encadré "Chronologie des rapports de l'OCDE sur le médicament ", p. 38] relatif aux publications de l'OCDE concernant le Boltanski, dans La Production de l'idéologie dominante, Paris, Démopolis, 2008 ; Voir également Vincent Gayon, "Lieux neutres en lutte. Consolidation inter-champs et
7. Au sens de Pierre Bourdieu, et Luc organisation multisectorielle internationale ", Culture \& Conflits, 108, 2017 p. 15-43.

8. Vincent Gayon, «Un atelier d'écriture internationale : I'OCDE au travail. Éléments de sociologie de la forme "rapport" ", Sociologie du travail, 51(3), 2009, p. 324-342.
9. Voir par exemple l'éditorial du Monde du 30 septembre 2014, "La vie n'a pas de prix, mais qui va payer?".

10. OCDE, Pharmaceutical Innovation and Access to Medicines, op. cit. 
du Centre de l'OCDE. Nous avons compilé et étudié l'ensemble des rapports de l'OCDE portant sur le prix des médicaments et plus largement sur la régulation pharmaceutique [voir encadré « Chronologie des rapports de l'OCDE sur le médicament », p. 38]. L’analyse de la controverse récente sur le Rapport 2 a supposé des observations au plus près de cette expertise en train de se faire. Nous avons assisté à la consultation des ONG avec l'OCDE (celle avec les entreprises nous a été refusée) ainsi qu'aux réunions entre la direction santé et les pays membres. Ces deux modalités d'enquête sont complétées par la conduite d'entretiens semi-directifs. Certains ont été anonymisés, les personnes ne souhaitant pas voir leur nom rendu public ou bien étant toujours en fonction.

\section{Une organisation internationale économique dans la mêlée de l'expertise sur le médicament}

Jusqu'au début des années 1990, le travail sur la santé à l'OCDE se limite à la comptabilisation des dépenses de santé de ses pays membres. En mettant en évidence l'augmentation de la part prise par les dépenses de santé dans les PIB des pays de l'OCDE, la cellule santé de l'Organisation s'assure le soutien financier de ses pays membres - en particulier les États-Unis - pour poursuivre le développement de sa base de données des dépenses de santé, ECO-Santé OCDE. En 2000, l'Organisation mondiale de la santé (OMS) publie un rapport classant l'ensemble des systèmes de santé des pays du monde selon un unique indice synthétique. Très critiqué par de nombreux économistes de la santé et experts gouvernementaux, ce rapport achève de délégitimer l'organisation onusienne, déjà mise en cause à la suite du mandat calamiteux de son directeur, le Dr Nakajima, des soupçons de corruption pesant sur sa direction, et des réticences de plusieurs pays industrialisés face à une organisation où les pays des Suds sont majoritaires ${ }^{11}$. La parution de ce rapport controversé aboutit à la redirection des fonds de certains pays comme les États-Unis, le Japon ou l'Australie vers l'OCDE « à la condition expresse qu'il n'y ait pas de classement $»^{12}$. Le groupe de travail sur la santé de l'OCDE voit dès lors ses capacités s'accroître et ses champs d'expertise s'élargir ${ }^{13}$.

\section{Les réserves étasuniennes sur l'opportunité d'un travail océdéen sur le médicament}

En dépit de sa position périphérique dans la Direction de l'emploi, du travail et des affaires sociales (DELSA) - dont la direction est systématiquement confiée à des économistes du travail, et non de la santé -, l'expertise sur la santé bénéficie d'un appui au plus haut niveau de l'Organisation : la santé est consacrée comme une «priorité centrale » en 2004, suscitant l'octroi de fonds spéciaux du Secrétariat général de l'Organisation, dirigé par le canadien Donald Johnson. Le groupe de travail sur la santé passe, dans le même temps, de la publication de statistiques sur les systèmes de santé des pays membres à la production de recommandations politiques ${ }^{14}$. Stimulée par la légitimation de sa base de données comparative des dépenses de santé, la division santé de l'OCDE emploie de plus en plus d'expertee-s permanentee's et s'affirme progressivement comme une référence dans le champ international de la réforme des politiques publiques de santée ${ }^{15}$.

À la $19^{\text {e }}$ réunion du comité santé en 2000 , un consultant de la division santé de l'Organisation, professeur d'évaluation pharmaco-économique à l'Université de Caroline du Sud, Michael Dickson, présente par exemple un projet relatif aux produits pharmaceutiques qui, déjà, laisse entrevoir des oppositions entre les pays membres. Le compte rendu de la réunion suivante du comité santé, en 2001, met en lumière le soutien au projet donné par plusieurs pays (notamment le Japon, le Royaume-Uni, la Suède et la Finlande) ${ }^{16}$, et l'opposition du plus gros bailleur de l'Organisation ${ }^{17}$ à la poursuite du travail. À la suite de la présentation du rapport, rédigé par Dickson et Jacobzone ${ }^{18}$, il y est précisé que la déléguée étasunienne s'est interrogée sur la relecture ou non de l'étude par des « experts médicaux appropriés ». Le compte rendu précise qu'elle demande, avec le soutien du délégué australien, que le rapport soit remanié « pour qu'il en ressorte que certaines des études actuelles visent plutôt
11. Frédéric Pierru, "Un classement révélateur. Les voies de l'économicisation de la doctrine de l'Organisation mondiale de la santé ", in Patrick Hassenteufel et Sylvie Hennion-Moreau (dir.), Concurrence et protection sociale en Europe, Rennes, PUR, 2004, p. 265-290.

12. $S G / A D H O C / H E A / M(2001) 1$, Compte rendu succinct de la 1 ère réunion du groupe ad hoc chargé du projet OCDE sur la santé, 26-27 mars 2001, p. 4.

13. Constantin Brissaud, "Des objectifs sanitaires à la rationalité budgétaire. La mise en marché des systèmes de santé saisie par leur quantification ", Revue française de socio-économie, 26, à paraître en juin 2021.

14. Constantin Brissaud, " La loi du (quasi-) marché. Innovations conceptuelles et légitimités expertes autour des soins de santé à l'OCDE (1977-2014) ", in Bruno Valat (dir.), Les Marchés de la santé en France et en Europe (XIXe-XXe siècles), Montpellier, Presses universitaires du Midi, à paraître fin 2020.

15. Frédéric Pierru et Marina Serré, «Les organisations internationales et la production d'un sens commun réformateur de la politique de protection maladie ", Lien social et Politiques, 45, 2001, p. 105-128. 16. Groupe de travail sur la politique sociale, compte rendu de la 19 e réunion, 16-17 octobre 2000, DEELSA/ELSA/ WP1/M(2000)1.

17. Les fonds de l'OCDE sont calculés au prorata du PIB des pays membres pour la part 1 du budget, la part récurrente. La part 2 du budget est constituée de contributions volontaires données par les pays membres pour que l'Organisation travaille sur un sujet particulier. 18. Stéphane Jacobzone (1964-) est un polytechnicien (X-ENSAE) passé par la Direction de la prévision du ministère de l'Économie et des Finances, puis par ECO (1997) et par la division santé de l'OCDE (2000-2002). Membre du collège des économistes de la santé, il est actuellement à la division GOV (réforme de l'administration publique) de l'OCDE. 


\section{Chronologie des rapports de l'OCDE sur le médicament}

2000 : Pharmaceutical Policies in OECD Countries. Reconciling Social and Industrial Goals. Ce rapport souligne l'augmentation des prix des produits pharmaceutiques dans les économies des pays de l'OCDE, et propose une revue des politiques nationales d'achat des médicaments innovants.

2003 : Survey of Pharmacoeconomic Assessment Activity in Eleven Countries. Le rapport plaide pour l'utilisation du Health Technologies Assessment (HTA) c'està-dire des outils économiques de calcul d'efficience pour les produits de santé, dont les médicaments, et le « partage d'expériences » entre les pays membres.

2008 : Les prix des médicaments sur un marché global : politiques et enjeux. Études de l'OCDE sur les politiques de santé. Le rapport montre que quelques médicaments concentrent la plus grande part des revenus des firmes pharmaceutiques, et que la régulation des prix ne conduit pas nécessairement à des prix moins élevés. II conclut sur l'utilité du HTA.

2013 : Value in Pharmaceutical Pricing. Cette note d'étude compare les procédures d'évaluation des innovations thérapeutiques dans 14 pays de l'OCDE afin de saisir comment le contrôle d'efficience ainsi que les études d'impact budgétaire sont pris en compte dans la définition de la valeur des traitements.
2016 : Pharmaceutical Expenditures and Policies. Past Trends and Future Challenges. Cette note de travail propose de détailler la structuration du marché du médicament en étudiant notamment les types de produits pharmaceutiques vendus. Pour la première fois, il traduit une inquiétude sur l'efficience des produits notant que certains produits " ne fournissent que des améliorations marginales ".

2017 (janvier) : New Health Technologies. Managing Access, Value and Sustainability. Ce rapport, que nous avons appelé " Rapport 1 » alerte sur un risque pour les finances des États membres si les prix des innovations ne sont pas contrôlés. II constitue un rapport offensif mettant en cause l'efficience des nouvelles thérapies contre le cancer et appelle à un "Rapport 2 " qui fournirait des préconisations en termes de politiques publiques.

2018 (novembre) : Pharmaceutical Innovation and Access to Medicines. Contrairement à ce qui était annoncé, ce "Rapport 2 » ne propose pas de préconisations fermes, mais appelle à une plus grande transparence dans les prix des médicaments et au renforcement de la coopération internationale et de la concurrence sur ce marché. 
à tester des hypothèses qu'à dégager des conclusions de fond. Les limites de l'analyse doivent être clairement mentionnées $»^{19}$.

Consignée dans le même compte rendu de séance, la réponse du Secrétariat témoigne de l'influence des États-Unis sur la direction des travaux pris dans le groupe de travail et sur leur capacité à faire inscrire noir sur blanc « les limites de l'analyse » :

«En réponse à l'interrogation des États-Unis,
le Secrétariat fait observer que cette étude a été
proposée au Groupe de travail en 2000 . [...] Le
rapport fera l'objet d'une version révisée dans
laquelle les sources de données seront précisées.
La formulation du rapport sera également adaptée
afin de mieux refléter les limites de l'analyse sur
laquelle reposent les résultats ${ }^{20}$. »

Entre-temps, le rapport sur « les produits pharmaceutiques » est devenu un rapport sur «l'utilisation des produits pharmaceutiques et les dépenses liées aux maladies cardio-vasculaires et aux accidents vasculaires cérébraux $»^{21}$. L'étendue du domaine d'étude du groupe de travail est ainsi circonscrite à des cas spécifiques, et dépouillée de toute visée généraliste. Qui plus est, ce rapport ne sera plus mentionné dans les comptes rendus des réunions du comité santé ultérieures. Stéphane Jacobzone, signe, seul, en 2000, un rapport sur les politiques du médicament dans les pays de l'OCDE ${ }^{22}$ sous-titré « Réconcilier les objectifs sociaux et industriels » (notre traduction), dans une collection relativement confidentielle : les Labour Markets and Social Policy Occasional Papers. Son rapport rappelle les investissements de recherche et développement consentis par l'industrie et se contente de conclure que «la régulation du secteur pharmaceutique doit réconcilier des objectifs sociaux et privés », ou encore que "les réformes des politiques du médicament doivent stimuler l'efficacité et préserver l'équité ». Il se garde de statuer sur les responsabilités respectives des producteurs et des payeurs, et en appelle au « (partage) des coûts de la R\&D au niveau international $»^{23}$.

L'expertise de l'OCDE sur le médicament fait face, depuis les origines, à l'hostilité du plus gros pays contributeur de l'Organisation : les États-Unis. Si ce pays a, historiquement, été parmi les premiers financeurs de la base de données des dépenses de santé construite par l'OCDE ${ }^{24}$, ses représentante-s ont, depuis, régulièrement limité les ambitions analytiques d'une division qui, de rapport en rapport, souligne l'inefficience des dépenses de santé extrêmement élevées des États-Unis. C'est ainsi que les rapports sur les médicaments produits par la division n'optent pas, jusqu'au milieu des années 2000, pour des recommandations politiques précises. Ce n'est qu'en 2005 qu'un nouveau rapport sur le sujet, est commandé à la division santé de l'OCDE. Financé par l'Australie, le Canada, la Finlande, la Hongrie et le Mexique, il est alors confié à deux économistes de la division santé de l'Organisation, dont Elizabeth Docteur, une « health policy analyst » étasunienne ayant déjà travaillé pour l'administration Clinton :

" J'ai dû me battre assez durement pour réaliser ce qui finit par être mon dernier gros projet à l'OCDE, qui a été un projet très difficile mais couronné de succès qui a donné lieu à $[\ldots]$ un rapport sur le prix des médicaments. C'était politiquement extrêmement sensible. [...] De nombreux pays avaient l'impression de s'être fait avoir par les États-Unis avec les accords commerciaux du GATT, et d'avoir accepté des termes très favorables à l'industrie pharmaceutique qui, à ce moment particulièrement... vous voyez... la plupart de ces compagnies était ostensiblement basées aux ÉtatsUnis et les États-Unis plaidaient pour elles, tentant de limiter le niveau de régulation des prix, en mettant des conditions extrêmement favorables en termes de droits de propriété, etc. [...] Il y avait donc, au mieux, une certaine ambivalence des États-Unis, et de temps en temps il y avait des pressions de la part des États-Unis pour diriger le travail de certaines manières et... enfin, c'était un projet vraiment très difficile ${ }^{25}$. »

Le «succès » du projet désigne avant tout la publication finale du rapport, dont le caractère " difficile" s'explique principalement par des raisons budgétaires. Pour engager un travail sur un thème nouveau, le Secrétariat doit, soit réunir des contributions volontaires (partie 2 du budget), soit recueillir l'assentiment de pays suffisamment puissants pour que le projet soit financé sur la partie 1 du budget (contributions régulières). Or, la part importante de la contribution des États-Unis ( $25 \%$ ) en fait un partenaire susceptible et écouté. En outre, le risque de représailles sur d'autres dossiers limite le soutien des pays à un travail dont les États-Unis souhaitent limiter la teneur critique : " (l'Australie, les Pays-Bas, la Suisse ou encore la France) ne voulaient pas faire partie des pays qui seraient sous pression des États-Unis en montrant qu'ils étaient des soutiens trop affirmés $»^{26}$.
19. Groupe de travail sur la politique sociale, compte rendu succinct de la $20^{\mathrm{e}}$ réunion, 24-26 octobre 2001, DEELSA/ ELSA/WP1/M(2001)1, p. 9.

20. Ibid., p. 9.

21. Ibid., p. 8.
22. Stéphane Jacobzone, Pharmaceutical Policies in OECD Countries: Reconciling Social and Industrial Goals, Paris, OCDE, 2000.

23. Ibid., p. 6.

24. Constantin Brissaud, "La genèse bureaucratique d'un lieu commun sectoriel : concurrences diplomatiques et soutiens institutionnels dans la production du premier rapport de l'OCDE sur les dépenses de santé (1985) ", Revue internationale de politique comparée, à paraître fin 2020.
25. Entretien avec Elizabeth Docteur, février 2017.

26. Entretien avec une économiste de la santé, membre de l'OCDE, février 2017. 
Une convergence de vue "pragmatique ": le département santé étasunien et l'industrie pharmaceutique

Les délégué·e·s étasunien-ne·s ne nient pas l'importance du sujet mais préfèrent le voir abordé dans les termes adoptés par l'industrie : l'innovation et son écosystème. C'est que ce secteur y est particulièrement stratégique : près de la moitié des emplois de l'industrie pharmaceutique des pays de l'OCDE sont en effet pourvus aux États-Unis, et environ $40 \%$ de la valeur ajoutée en R\&D y est investie. Les liens entre les instances de régulation et l'industrie pharmaceutique sont par ailleurs particulièrement étroits aux États-Unis ce qui renforce l'alignement des représentant-e·s étasunien-ne·s à l'OCDE sur les positions des organismes en charge de représenter les industries ${ }^{27}$ comme en atteste le compte rendu de la réunion du groupe de travail de novembre 2002.

Après que plusieurs pays ont fait état de la nécessité d'améliorer la coopération sur l'évaluation pharmacoéconomique ${ }^{28}$ - le délégué australien la présentant même comme « le Graal »-, le délégué étasunien est le seul à exprimer un avis plus réservé : « (L'évaluation pharmaco-économique) ne devrait pas être utilisée pour contenir les coûts car les entreprises pharmaceutiques ont besoin de retours adéquats sur leurs investissements en R\&D si l'on veut voir l'innovation se poursuivre. Il est important de ne pas freiner l'innovation. $»^{29} \mathrm{Ce}$ positionnement est partagé par les représentante-s des industries à l'OCDE (le Business and Industry Advisory Committee - le BIAC). Dirigé, pour sa «Task Force Santé », par Alain Sommer, un médecin diplômé de Sciences Po Paris et HEC, ancien consultant pour le cabinet Andersen et responsable, au moment de la réunion, du déploiement de services financiers pour Arcelor, le BIAC prône une ligne en phase avec le point de vue étasunien. Le thème de «l'innovation » permet de déplacer le problème des prix vers les conditions d'approfondissement de la recherche et du développement, et s'avère donc favorable aux industriels du médicament comme aux pays dont ils sont les hôtes.

Les représentant·e·s des États-Unis à l'OCDE et les membres du BIAC adoptent, au cours des années 2000, une stratégie convergente de lobbying, consistant à faire rencontrer leurs émissaires, tous deux étasuniens, à l'auteure principale du rapport de 2008. Le premier est John Hoff, alors « Deputy Assistant Secretary » pour la planification et l'évaluation au département de la santé et des services sociaux des États-Unis d'Amérique où il travaillait principalement sur les politiques de soins de longue durée, les invalides et les personnes âgées. Diplômé en droit de l'Université Harvard, il cofonde le Galen Institute, un think tank libertarien sur les politiques de santé, et est, depuis 2010, particulièrement mobilisé contre l'Obamacare dans des articles qu'il écrit pour l'American Enterprise Institute, un autre think tank étasunien proche du Parti républicain. John Hoff est nommé à temps plein à Paris au moment même où Elizabeth Docteur se voit accorder le mandat pour travailler sur les modalités de fixation des prix des médicaments. Outre cette dernière, il rencontre alors régulièrement le Secrétaire général de l'OCDE. Pour John Cohrssen, un autre émissaire de nationalité étasunienne mais travaillant, lui, pour le BIAC, Hoff a d'ailleurs été expressément envoyé par le gouvernement des États-Unis pour limiter les perspectives régulatrices de l'OCDE sur le médicament. L'émissaire du gouvernement étasunien et celui du BIAC défendent les mêmes perspectives «pro-market $»^{30}$ sur le médicament. « Pourquoi croyez-vous que l'industrie pharmaceutique envoyait John Cohrssen pour déjeuner avec moi ?", questionne ainsi Elizabeth Docteur.

Indice de l'importance des préconisations océdéennes en la matière, les experts travaillant sur le rapport font donc face à des pratiques de lobbying plus ou moins agressives. John Hoff, en particulier, a précisément été envoyé par le gouvernement étasunien pour critiquer régulièrement, en séance et auprès du Secrétaire général, les rapports produits par la division santé de l'OCDE. Tout comme les experts du BIAC, sa tâche est de demander à ce que les études de politiques publiques particulières soient retirées, au profit d'analyses quantitatives qui, seules, pourraient permettre de tirer des conclusions ${ }^{31}$. Mais outre les tentatives d'influence des États et des représentant·e-s de l'industrie, les experte-s de la division santé doivent, à partir de 2001, compter avec la concurrence de la division des affaires économiques (ECO).

\section{Concurrence intra-bureaucratique pour l'expertise sur la santé}

Au sein de l'OCDE, l'émergence de la santé parmi les objets de travail légitimes de l'Organisation suscite d'abord l'intérêt du groupe de travail PUMA (Public Management) à partir de 1995, puis de la Direction ECO (affaires économiques). Si PUMA, « certainement
28. Sur l'évaluation médico-économique, Grenoble, PUG, 2020. voir Daniel Benamouzig, La Santé au miroir de l'économie, Paris, PUF, 2005 et Cyri Benoît, Réguler l'accès aux médicaments,
29. DELSA/ELSA/WP $1 / M(2002) 2$,

" compte rendu succinct de la $22^{\mathrm{e}}$ réunion,

18-20 novembre 2002 », p. 5.
30. Comme ils la caractérisent tous les deux en entretiens (septembre 2018).

31. Entretien avec John Hoff, septembre 2018. 
le réseau international le plus influent dans (la) diffusion (du New Public Management) $»^{32}$ prend en charge en priorité les questions de gestion hospitalière, les « documents de travail» de la division ECO sont, eux, nettement plus englobants. Appliquant les méthodes de la micro-économie standard, ils prennent de plus en plus pour objet la santé à partir de 2000, y « (introduisant) des mécanismes d'incitation qui avaient fait leurs preuves dans le secteur des entreprises ${ }^{33}$ indépendamment de la spécificité - pourtant régulièrement rappelée par les économistes de la santé ${ }^{34}-$ du secteur des soins de santé. Dans les rapports d'ECO ou PUMA, les hôpitaux doivent, sous la pression de la concurrence, adopter des « ajustements structurels [...] les poussant au changement $»^{35}$, et, à ECO, le benchmarking des systèmes de santé est toujours la clé pour assurer une plus grande efficience des dépenses ${ }^{36}$. Si les rapports d'ECO ne ciblent jamais le médicament en tant que tel, ils proposent en revanche de penser les systèmes de santé comme d'autres types de dépenses publiques. Le rapport d'ECO sur le système de santé étasunien reprend par exemple les récriminations routinières de son ou sa représentant $\cdot e$ - ainsi que des représentant·e·s $\mathrm{du}$ BIAC - envers les autres pays. Pour ces dernier.e's en effet, le contrôle des prix des médicaments dans de nombreux pays obligerait les industriels à réaliser leur marge sur le seul marché solvable et peu régulé, le marché étasunien. En ce sens, les autres pays agiraient comme des "passagers clandestins », en laissant aux États-Unien'ne-s le soin de payer pour l'innovation ${ }^{37}$.

Structurellement dominée par la Direction des affaires économiques (ECO) à l'intérieur de l'OCDE, la division santé doit savoir contenter ses bailleurs pour maintenir des fonds majoritairement issus de la part 2, les contributions volontaires des États (et non de la part 1 du budget, calculée en fonction du PIB des pays membres) interdisant ce faisant des prises de position trop évidemment hostiles aux industriels du médicament.

\section{Vers une expertise océdéenne critique de l'industrie pharmaceutique?}

À partir de 2013, la division santé de l'OCDE travaille à la production d'une expertise renouvelée sur le marché pharmaceutique $^{38}$. Plusieurs travaux universitaires et rapports internationaux ont en effet pointé l'inefficience des dépenses de santé - notamment concernant les médicaments - aux États-Unis, et, plus généralement, l'industrie pharmaceutique se trouve mise en cause. Si la division tente d'infléchir ses positions historiques en mettant en lumière des taux de rentabilité élevés et des niveaux d'efficience insuffisants pour les « innovations thérapeutiques », cette tentative - dont témoigne le contenu du rapport de janvier 2017 (le « Rapport $1 »)$ - s'inscrit dans une période où les prix des thérapies innovantes, notamment contre le cancer, connaissent une augmentation saisissante qui oblige les pays de l'OCDE à se pencher sur la question.

\section{De l'hépatite $C$ au Cancer :}

\section{les pays de l'OCDE face à la crise des prix}

Les prix du médicament étaient auparavant principalement problématiques pour les pays des Suds, mais l'affaire du Sovaldi, un médicament contre l'hépatite C, étend à partir de 2014 la question du "rationnement » aux pays de l'OCDE. La controverse ne touche plus seulement des traitements contre le sida ${ }^{39}$, mais une multitude de pathologies, des hépatites au cancer, et les montants en jeu sont sans commune mesure avec les années 1980 : non plus 10000 dollars pour un traitement mais 100 000, 200000 dollars voire au-delà ${ }^{40}$. Amplement documentée dans de nombreux rapports, cette explosion des prix du médicament ne devient politiquement inquiétante pour les pays de l'OCDE que lorsqu'elle a un effet sur le budget global des dépenses de santé. La controverse sur le Sovaldi a constitué sur ce point un événement, le Sénat étasunien allant jusqu'à ouvrir une enquête sur la façon dont le laboratoire, Gilead, a fixé le prix du médicament [voir encadré « Le Sénat étasunien enquête sur le prix du Sovaldi », p. 42 et illustration, p. 43].

Le prix des médicaments « innovants » est constitué en problème public dans les pays riches au cours de l'année 2014 non seulement parce qu'il est saisi par une autorité publique (par exemple aux États-Unis le Sénat ou en France le ministère de la Santé) mais aussi car il devient l'objet d'une mobilisation visant à le mettre à l'agenda, les ONG jouant dans ce processus un rôle d'entrepreneurs de morale ${ }^{41}$. Comme dans d'autres pays, Médecins du Monde lance par exemple
32. Philippe Bezes, dans «État, experts et savoirs néo-managériaux. Les producteurs et diffuseurs du New Public Management en France depuis les années 1970 ", Actes de la recherche en sciences sociales, 193, 2012, p. 16-37 et en particulier p. 30. 33. OCDE, À la recherche de mécanismes de marché : les systèmes de santé au Canada, en Islande et au Royaume-Uni, Paris, OCDE, 1995, p. 3.
34. Voir Kenneth J. Arrow, "Uncertainty and the welfare economics of medical care", The American Economic Review, 53(5), 1963, p. 941-973.

35. OCDE, À la recherche de mécanismes de marché..., op. cit., p. 21.

36. Par exemple OCDE, Spending Efficiency in the OECD: Benchmarking Health Care, Education and General Administration, 2016, p. 12.
37. Paul Roderick Gregory, "Obama care will end drug advances and Europe's free ride (unless China steps in)", Forbes, $1^{\text {er juil- }}$ let 2012 ; Health Care Reform in the United States, Paris, OECD Publishing, 2009, p. 15 (notre traduction).

38. Valérie Paris et Annalisa Belloni, Value in Pharmaceutical Pricing, OECD Health Working Papers, Paris, 2013.

39. Sébastien Dalgalarrondo, Sida: la course aux molécules, Paris, Éd. de l'EHESS, 2004, p. 43.

40. John Lauerman et James Paton, "Novartis's $\$ 475,000$ price on cancer therapy meets resistance", Bloomberg.com, 22 septembre 2017.

41. Howard Becker, Social Problems: A Modern Approach, New York, John Wyler, 1966. 


\section{Le Sénat étasunien enquête sur le prix du Sovaldi}

En 2014, une enquête de 18 mois du comité des finances des États-Unis d'Amérique conduite par les sénateurs démocrate de l'Oregon Ron Wyden et républicain de l'lowa Charles Grassley est ouverte sur l'impact budgétaire du Sovaldi, un médicament contre l'hépatite $\mathrm{C}$ dont la prise en charge publique conduit la moitié des programmes Medicaid à restreindre l'accès du médicament aux populations les plus malades ${ }^{1}$ : les dépenses de médicament contre l'Hépatite C du US Bureau of Prisons augmentent par exemple de 14 \% en 2014 alors que le nombre de patient.e.s traité.e.s baisse lui de $52 \%$. Le point central de leur enquête est que, d'après les documents internes à l'entreprise, le prix du médicament n'a pas été fixé à partir des coûts de recherche et développement mais sur la base d'un calcul visant à maximiser le prix que le pays payeur serait prêt à accepter. Cette affaire a trois conséquences. Premièrement, Gilead et son médicament deviennent l'incarnation d'une industrie pharmaceutique " cupide $»^{2}$, financiarisée et indifférente aux enjeux sociaux. Deuxièmement, le rapport du Sénat devient pour certain.e.s militant.e.s l'incarnation d'un État qui se saisit d'un problème et convoque un laboratoire mondialement connu (un.e de nos interviewé.e.s, membre d'une ONG, parle d'une
" bible » qu'il.elle souhaite traduire et envoyer à tous les parlementaires français.e.s). Ce rapport constitue de fait un document unique en son genre puisque aucune enquête de ce type n'a jamais été publiée. Enfin, le rapport généralise le problème au-delà de l'hépatite $C$ : «Bien que notre enquête se concentre sur un médicament - un médicament certes particulier - nous pensons qu'elle est d'importance parce que si les États-Unis souhaitent guérir d'Alzheimer, du cancer, des diabètes et du Sida dans les temps à venir, ces médicaments ne seront pas accessibles et bien trop chers pour des millions d'Américains ${ }^{3}$."

1. Orrin G. Hatch et Ron Wyden, The Price of Sovaldi and its Impact on the US Health Care System, 2015, p. 99.

2. William Lazonick, Matt Hopkins, Ken Jacobson, Mustafa Erdem Sakinç et Öner Tulum, "US pharma's business model. Why it is broken, and how it can be fixed", in David Tyfield, Rebecca Lave, Samuel Randalls et Charles Thorpe (dir.), The Routledge Handbook of the Political Economy of Science, Londres, Routledge, 2017, p. 83-100. Gilead n'en est pas à son premier coup d'éclat (voir Sébastien Dalgalarrondo, Sida : la course aux molécules, Paris, Éd. de l'EHESS, 2004).

3. "Wyden press conference remarks on investigation into Gilead's pricing of hepatitis drug Sovaldi", Press Conference, $1^{\text {er }}$ décembre 2015, en ligne. Notre traduction. 

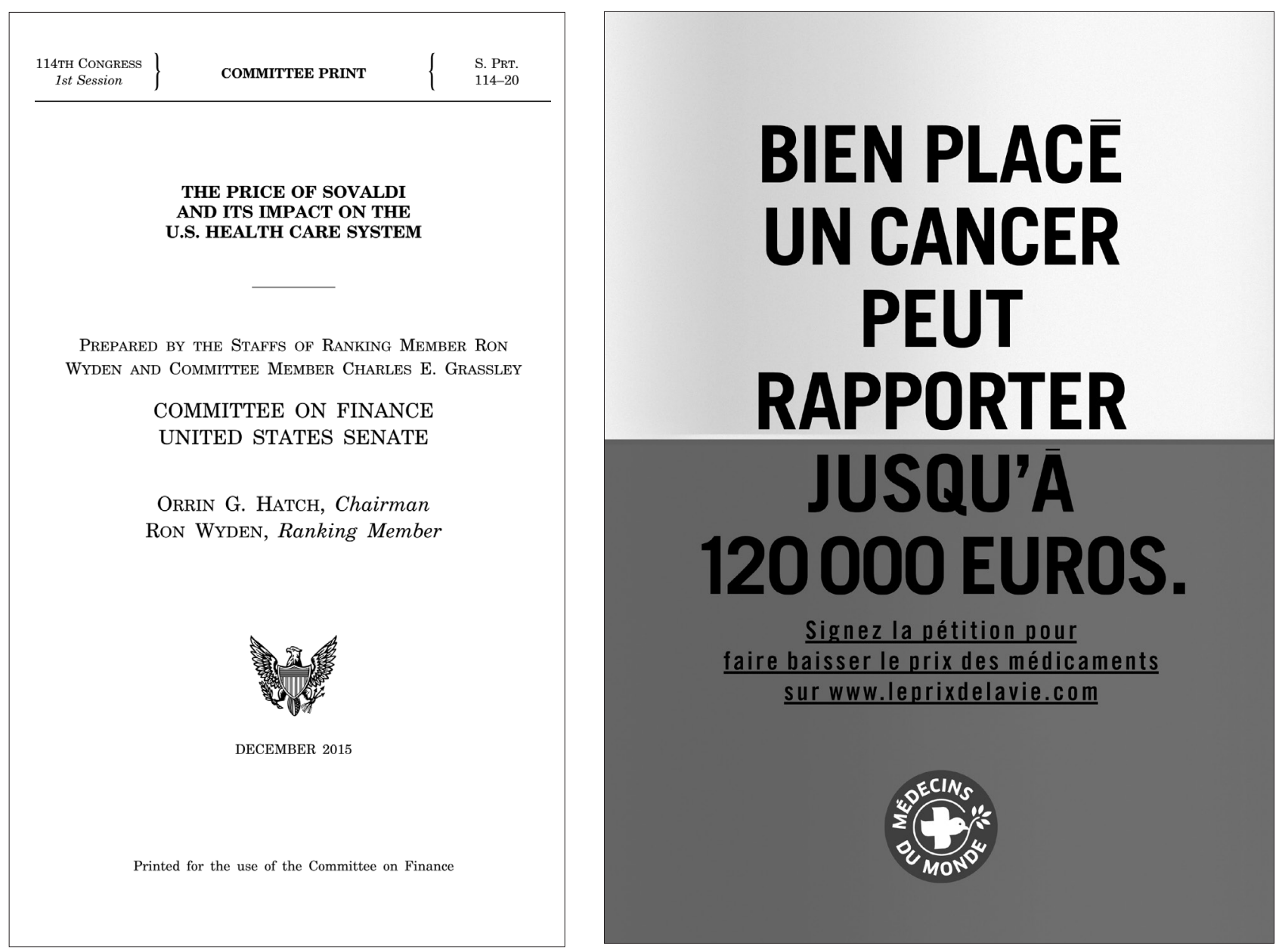

COUVERTURE DU RAPPORT " The Price of Sovaldi and its Impact on the US Health Care System " pour le comité des finances du Sénat américain, décembre 2015.

AFFICHE DE MÉDECINS DU MONDE dans le cadre de la campagne « Le prix de la vie », 2016. 


\title{
ANNEX 3: LIST OF CONSULTATIONS AND MEETINGS
}

\author{
Sustainable Access to Innovative Therapies \\ CONSULTATIONS WITH STAKEHOLDERS
}

2016

\begin{tabular}{|l|l}
\hline 1-3rd November & $\begin{array}{l}\text { Consultations at World Cancer Congress with patient groups, providers and } \\
\text { NGOs }\end{array}$ \\
\hline 5 December & BIAC Consultation \\
\hline 6 December & 1st High Level Expert Group \\
\hline 13 March - 1 May & Online consultation \\
\hline 9 May & Workshop for National Experts \\
\hline 15 May & 2nd High Level Expert Group \\
\hline 16 May & Technical consultation with BIAC \\
\hline 20 June & Civil Society Consultation \\
\hline 28 June & BIAC Consultation \\
\hline 8 September & TUAC Consultation \\
\hline 25 October & United States Council for International Business \\
\hline 26 October & United States National Association of Manufacturers \\
\hline 26 October & America's Health Insurance Plans \\
\hline 27 October & United States Pharmaceutical Care Management Association \\
\hline 27 October & United States Association for accessible medicines \\
\hline
\end{tabular}

CONSULTATION AVEC LES PARTIES prenantes pour la rédaction du « Rapport 2 ".

Source : DELSA/HEA(2017)17/ANN, Sustainable access to Innovative Therapies - Draft Report Annex, présenté à la $22^{\mathrm{e}}$ session du comité santé des 11 et 12 décembre 2017, p. 146. 
en France, après plusieurs tentatives de négociations avec le ministère de la Santé une campagne dénonçant des prix exorbitants et demandant à l'État de contrôler ces augmentations ${ }^{42}$.

Le problème fait également son apparition dans les discussions et les conférences internationales à un très haut niveau politique. Dans le communiqué final du sommet de Kobé des 11 et 12 septembre 2016, les ministres de la Santé du G7 évoquent ainsi quatre points. Les trois premiers concernent le développement de la santé globale, la couverture santé universelle, la résistance aux antimicrobiens. Le quatrième point fait écho à la crise du Sovaldi et à la question récurrente des prix des innovations thérapeutiques. Le G7 souhaite réfléchir à des modalités de régulation permettant, tout en préservant un « environnement positif pour l'innovation ", de garantir l'accès au médicament et la "soutenabilité des systèmes de santé ». Le communiqué se termine ainsi : "Nous souhaitons prendre en compte l'expertise de l'OCDE sur ce sujet et l'évoquerons à sa prochaine réunion des ministres de la Santé ${ }^{43}$. Face à la publicisation du problème du prix des médicaments, se tient les 16 et 17 janvier 2017 la réunion ministérielle, annoncée par le G7, au siège de l'OCDE à Paris. Or l'organisation n'a pas attendu une saisine de la ministre française ${ }^{44}$ ni le communiqué de Kobé pour plancher sur le sujet.

\section{Une tentative de durcissement des recommandations}

En 2014, la division santé de l'OCDE a déjà organisé une série de séminaires sur le thème de la régulation pharmaceutique. L'enjeu de ces réunions est d'échanger avec des représentant-e-s des pays membres afin qu'ils fassent part des difficultés qu'ils rencontrent en matière de fixation des prix. Le problème s'avère alors commun à l'ensemble des pays membres. Fait nouveau au regard du rapport précédent, ce processus incorpore des consultations avec des ONG qui participent à la définition du problème et des solutions envisageables.

Le Rapport 1 de janvier 2017, New Health Technologies: Managing Access, Value and Sustainability, vise à la fois à dresser un état des lieux, à formuler un « diagnostic » et à enclencher une réflexion en termes de régulation possible. L'OCDE y affirme, par exemple, observer que « de nombreux produits en oncologie sont financés alors qu'ils ne sont pas efficients $»^{45}$, une prise de position qui dans le langage tempéré des institutions internationales s'avère particulièrement critique. Ainsi, l'augmentation des prix des innovations est confrontée à l'évaluation de la qualité thérapeutique de ces dernières : « Le prix des technologies doit refléter les bénéfices que celles-ci apportent concrètement en termes de santé par rapport à d'autres possibilités, et ce prix doit être ajusté en fonction des preuves de leur impact réel. Les organismes payeurs doivent être dotés des pouvoirs nécessaires pour en ajuster les prix et cesser de les rembourser en cas d'inefficacité $»^{46}$. Le Rapport 1 de janvier 2017 constitue un point de fixation des alternatives sur le prix du médicament. Comme auparavant, la doctrine de la division santé de l'OCDE peut être résumée par la conciliation de trois objectifs : s'inscrire dans une logique de marché et d'incitation à l'innovation ; empêcher des phénomènes de blocages en matière de mise sur le marché des médicaments ; limiter le montant des dépenses publiques de santé. Ce rapport de janvier 2017 (le Rapport 1), considéré comme une étape avant la publication du rapport définitif initialement prévu pour la fin de la même année (le Rapport 2), se distingue pourtant des précédents par un ton plus offensif, questionnant le niveau des prix, pointant les responsabilités des laboratoires pharmaceutiques et plaidant pour des réformes radicales du mode de fixation des prix.

D'un bout à l'autre de la chaîne de production du médicament, le rapport souligne l'ambiguité des laboratoires : les incitations en $R \& D$ pour les médicaments orphelins sont "détournées ${ }^{47}$, "les renseignements communiqués par les entreprises chargées de procéder aux essais cliniques sont incomplets et biaisés de manière à donner des résultats positifs ", les produits sont comparés à des placebo au lieu d'être comparés aux médicaments existants, ou encore la population-test est « sélectionnée » par le laboratoire ${ }^{48}$. Si elles sont évoquées sur le ton qui sied à un rapport d'organisation internationale, ces réserves sont néanmoins accablantes. D'autre part, le rapport pointe que l'industrie pharmaceutique est en perte de vitesse : «Le rapport coût-bénéfice (des nouvelles thérapeutiques) tend à se révéler nettement moins favorable (que par le passé) » ${ }^{49}$ et « (les médicaments utilisés pour traiter des affections et/ou maladies particulièrement graves pour lesquelles aucun traitement n'est disponible) ne présentent pas un bon rapport coût-efficacité au regard des seuils habituels $»^{50}$. Le rapport plaide ainsi pour « un effort de transparence et de coopération entre les payeurs et des achats groupés internationaux » tout en spécifiant que « les accords de performance (qui sont le mode de tarification défendu par les industriels) doivent être utilisés avec parcimonie $\aleph^{51}$. D’une manière générale, le 
pouvoir de négociation des prix est considéré comme trop favorable aux entreprises du médicament, et donc à rééquilibrer en faveur des États.

\section{D'un rapport à l'autre : la neutralisation de la critique}

Du Rapport 1 au Rapport 2, la division santé de l'OCDE organise plusieurs consultations, avec les représentant e-s des syndicats, les représentant e.s de l'industrie (le BIAC), celles et ceux des pays membres et des ONG [voir illustration, p. 43]. Alors que les rédacteurs et rédactrices envisagent d'inscrire dans le rapport des pistes de régulation contraignantes pour les industries, ceux-ci se heurtent au lobbying du BIAC et de certains pays qui cherchent à promouvoir une piste de régulation alternative, relativement floue et leur étant favorable : le " paiement à la valeur».

\section{Les ressources limitées des ONG et des syndicats}

Du point de vue de la méthode, la division santé décide d'opérer des modifications dans la production du Rapport 2. Alors que les consultations fonctionnent en principe sur un mode binaire avec les représentants des syndicats et du patronat (avec respectivement le TUAC - le Trade Union Advisory Committee - et le BIAC), la division santé décide d'organiser une journée de consultation avec les ONG spécialisées dans l'accès au médicament ${ }^{52}$. Ainsi, le 20 juin 2017, DELSA accueille à l'OCDE, dans le $16^{\mathrm{e}}$ arrondissement de Paris, de $13 \mathrm{~h} 30$ à 18 h, une trentaine de personnes pour une "Civil Society Consultation » qui vise à présenter le Rapport 1 , et à recueillir des retours sur les options politiques proposées ${ }^{53}$. Dans la salle, 38 personnes $^{54}$ repré- $^{-}$ sentent principalement des associations ou des ONG de différents pays de l'OCDE.

Certaines options, déjà évoquées dans le Rapport 1 , concernent les coopérations entre pays pour peser dans les négociations avec les industriels ${ }^{55}$ quand d'autres cherchent à promouvoir la transparence sur la façon dont les prix sont fixés. Héritée des mobilisations pour l'accès aux trithérapies, la position des
ONG en matière d'accès au médicament se focalise sur la question de la brevetabilité. Insistant sur la tension entre propriété des inventions et santé publique ${ }^{56}$, les ONG dénoncent majoritairement un régime juridique issu d'accords de l'OMC pouvant entraver la provision de soins ${ }^{57}$.

Durant la consultation, les ONG insistent sur la nécessité de "modèles alternatifs » aux brevets tels qu'ils fonctionnent aujourd'hui, en mobilisant notamment la «licence d'office », qui permet à un État d'exploiter lui-même le brevet, c'est-à-dire de produire ou faire produire le médicament, lorsque des médicaments brevetés « ne sont mis à la disposition du public qu'en quantité ou qualité insuffisante ou à des prix anormalement élevés $»^{58}$. En d'autres termes, il s'agirait de faire primer la santé publique sur les retours sur investissements des industriels du médicament.

Ces positions, les acteurs en présence ont l'habitude de les défendre dans d'autres arènes internationales, Union européenne et Organisation mondiale de la santé (OMS) entre autres, mais c'est la première fois qu'ils se retrouvent consultés ainsi par l'OCDE. Allant jusqu'à craindre une instrumentalisation de la forme " consultation », leur·e·s déléguéee·s avouent pour l'heure ne pas considérer l'OCDE comme un lieu majeur de leur opération de plaidoyer :

\begin{abstract}
On ne mange pas, on ne dort pas OCDE pour être honnête. L'OCDE, c'est intéressant, c'est une bonne chose qu'ils aient rendu ce rapport (le Rapport 1). Le fait qu'ils formulent tout ça c'est une bonne chose mais on est à un niveau et à des moyens où voir avec la Commission européenne, rencontrer et comprendre comment bosse l'INPI ${ }^{59}$, dialoguer avec le ministère, voir ce qui va passer en projet de loi de finance, c'est bien au-dessus de la pile que l'OCDE ${ }^{60}$.
\end{abstract}

L'influence limitée des ONG sur l'OCDE trouve trois explications. D'abord le faible lien de l'OCDE et des ONG dans le domaine de la santé a un impact sur l'engagement de ces dernières dans cette arène. Deuxièmement, la technicité d'un sujet dont ces organisations commencent seulement à se saisir : le prix du médicament suppose d'entrer au cœur des instruments d'évaluation économiques et thérapeutiques, ce que n'ont pas encore
52. II s'est agi d'organisations canadiennes (Save Your Skin Foundation), suisses (Public Eye), françaises (Ligue contre le cancer, Médecins du monde, AIDES, Médecins sans frontières) étasuniennes (Knowledge Ecology International) et européennes (European Cancer Organisation, European Patients' Forum, European Alliance for responsible $R \& D$ and Affordable medicines, European Public Health Alliance, etc.).

53. OECD, "OECD project on sustain- able access to innovative therapies: civil society consultation", 20 juin 2017. Notre traduction.

54. D'après la liste des inscrit.e.s fournie au début de la réunion. Nous avons comptabilisé entre 30 et 40 personnes.

55. C'est ce qu'ont fait très récemment les pays du Benelux et l'Autriche en mettant sur pied une collaboration appelée BeNeLuxA dont l'objectif est " d'assurer un accès soutenable à l'innovation médicale pour des coûts abordables pour les patients ". 56. Maurice Cassier, "Brevets pharmaceutiques et santé publique en France : opposition et dispositifs spécifiques d'appropriation des médicaments entre 1791 et 2004 ", Entreprises et histoire, 36(2), 2004, p. 29-47.

57. Gaëlle Krikorian et Amy Kapczynski (dir.), Access to Knowledge in the Age of Intellectual Property, New York, Zone Books, 2010.
58. Article L.613-16 du Code de la propriété intellectuelle ; voir également M. Cassier, op. cit., notamment p. 29.

59. Institut national de la propriété intellectuelle.

60. Entretien avec un.e salarié.e d'une ONG présent.e à la consultation, septembre 2017. 
fait la très grande majorité d'entre elles ${ }^{61}$. Enfin, les moyens limités des ONG les contraignent à arbitrer entre les différentes arènes à investir en termes de plaidoyer dès lors qu'elles n'ont pas encore la « force de frappe » nécessaire.

Sur le premier point, il est à relever que les évolutions qu'a connues le champ de la régulation du médicament depuis 2018 ne se sont pas jouées à l'OCDE mais à l'OMS, organisation que connaissent davantage les ONG et où chaque pays dispose d'une voix. La résolution adoptée le 28 mai 2019 à l'OMS a néanmoins, de la même façon que pour le Rapport 2 de l'OCDE, fait l'objet d'attaques multiples et répétées de la part de pays membres comme le Royaume-Uni et des représentante-s de l'industrie pharmaceutique. Mais là, les ONG sont parvenues à contraindre l'OMS - qui, en tant qu'agence de l'ONU, compte de nombreux pays des Suds économiques - de voter cette résolution ${ }^{62}$ qui « exhorte les États membres à améliorer l'échange public d'informations sur les prix effectivement payés par les gouvernements et d'autres acheteurs de produits sanitaires, et à accroître la transparence sur les brevets pharmaceutiques, les résultats des essais cliniques et les autres déterminants des prix le long de la chaîne de valeur, du laboratoire au patient $»^{63}$.

Si les ONG sont mobilisées pour la première fois dans l'histoire de la division santé à l'OCDE entre le Rapport 1 et le Rapport 2, ce n'est en théorie pas à elles que revient le rôle de représentante de la société civile. Cette représentation est historiquement assurée par les syndicats : aux consultations du BIAC - la représentation des industriels à l'OCDE - doivent en principe répondre celles du TUAC - les syndicats. Or, tout comme pour les ONG, le TUAC ne dispose ni de capacités d'expertise, ni de moyens comparables au BIAC. Ainsi, le TUAC ne s'intéresse que depuis peu au domaine du médicament et le délégué en charge de ces questions, Ronald Jansen a dû, pour rendre compte du travail actuel de l'OCDE, faire appel à des contacts noués au temps où il travaillait pour les syndicats chrétiens belges, de sorte que le TUAC ne dispose pas de capacités d'analyse autonomes suffisantes sur ce sujet $^{64}$. Si les représentations du BIAC et du TUAC à l'OCDE sont en principe symétriques, le différentiel de moyens est tel que les initiatives de l'un ne peuvent pratiquement jamais être suivies par l'autre. Et pour cause, le délégué du TUAC sur le dossier médicaments est chargé de plusieurs autres dossiers importants, comme les politiques d'emploi qui constituent le cour de son domaine de compétence. Il n’a commencé à suivre les dossiers santé qu'en janvier 2017, quand, de son côté, le BIAC dispose d'une « task force » expérimentée sur le dossier santé, et peut mobiliser jusqu'à quatre agents pour se déplacer aux réunions du comité.

\section{Le BIAC et l'annihilation de la critique}

Illustration des capacités d'expertise différenciée du TUAC et du BIAC, le Rapport 1 fait l'objet, de la part de la représentation des industriels, d'un contrerapport de 120 pages que ses experts apportent lors de la consultation avec les experte-s de la division santé le 28 juin. Ce document souligne la contribution des industriels du médicament au bien public justifiant les incitations financières à l'innovation que doivent garantir les États, et accuse la possibilité du recours à la licence d'office de diminuer les incitations à l'innovation, fragilisant ce faisant les chances de découverte de nouveaux médicaments innovants.

Lors des consultations, les expert·e-s du BIAC cherchent à écarter certaines références scientifiques. Or ces articles ont été recommandés par un groupe d'experte.s « de haut niveau » mis en place par les membres de la division santé en charge du dossier médicaments pour l'aider à réfléchir (le « High Level Expert Group » mentionné dans l'illustration 3) [voir p. 44], et ajouter leur légitimité à celle de l'Organisation dans ce dossier miné. Parmi ces experte•s, on trouve ainsi Peter Bach, un épidémiologiste étasunien fréquemment présent dans les colonnes du New York Times, Brigitte Dormont, économiste de la santé à Paris-Dauphine, Suzanne Hill, Peter Smith ou encore Joseph Stiglitz. Si ces expertees n'ont jamais pris position pour une réforme du droit des brevets ou pour des mécanismes comme la licence d'office, ils sont en revanche connus pour leurs positions « régulatrices ». Peter Bach, chercheur au Memorial Sloan Kettering est par exemple l'auteur de nombreux travaux dénonçant des prix sans rapport avec l'efficacité des médicaments. Certains membres de ce groupe vont recommander l'intégration dans le rapport d'un article, publié dans l'American Economic Review démontrant que, dans le domaine du cancer, le modèle économique et financier de l'industrie pharmaceutique détourne les laboratoires de la recherche d'innovations de long terme, les conduisant à cibler des produits de niches plus rentables ${ }^{65}$. Point d'appui potentiel à la critique de l'industrie pharmaceutique et des prix qu'elle pratique,
61. Certaines organisations européennes comme la European Public Health Alliance sont, elles plus, avancées sur ces sujets. 62. Pauline Londeix et Jérôme Martin, "Prix des médicaments : dans l'arène du débat ", Vacarmes, 88, 2019, p. 60-67. 63. Communiqué en ligne sur le site de l'OMS. 64. Entretien avec Ronald Janssen, avril 2018.
65. Eric Budish, Benjamin N. Roin et Heidi Williams, "Do firms underinvest in long-term research? Evidence from cancer clinical trials", The American Economic Review,

105(7), 2015, p. 2044-2085.

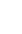


cet article, malgré sa parution dans une des plus prestigieuses revues d'économie mondiale, est retiré des versions de travail préalables ${ }^{66}$. Cette annihilation de la critique par la suppression d'articles scientifiques illustre cependant la capacité d'influence du BIAC, l'article étant finalement absent du Rapport 2.

Ce travail d'influence de l'écriture par le choix des références, de même que le refus de l'inscription de propositions comme l'utilisation de la licence d'office, vont de pair avec une activité de lobbying soutenue de la part du BIAC s'étendant au-delà des consultations mises en place par la division santé, et surpassant donc de beaucoup la capacité d'influence du TUAC. $\mathrm{Au}$ lendemain de la réunion ministérielle d'octobre 2017, le BIAC organisait par exemple un grand «Forum sur la santé, la croissance et la productivité », où étaient invité·e·s des hauts fonctionnaires de l'OCDE, des chefs d'entreprise et des officiel·le's « de haut niveau ». À cette occasion, Thomas Cueni, directeur général de l'IFPMA (la puissante International Federation of Pharmaceutical Manufacturers $^{67}$ ) a notamment pu présenter le « rôle proactif du secteur privé dans une meilleure santé, croissance et productivité ${ }^{68}$.

\section{Des market failures aux policy failures : des pays membres divisés}

L'observation de la présentation du pré-rapport lors du comité santé de juin 2017 permet de saisir les interactions entre les représentants du comité santé de l'OCDE et les délégué·e-s des pays lors de la réception du rapport. Par rapport aux autres réunions de ce type, celle-ci s'avère très suivie, et par des délégué·e·s « de haut niveau ». Les États-Unis sont par exemple représentés par Peter Schmeissner ${ }^{69}$, directeur Europe et Eurasie au département de la santé et des services sociaux des États-Unis d'Amérique, et le directeur du département Employment, Labour and Social Affairs (DELSA) de l'OCDE - dont dépend la division santé - est également présent.

Dès le début de la réunion, le BIAC distribue à la quarantaine de délégué·e·s présents une feuille rectoverso en papier glacé reprenant les principales récriminations contre le Rapport 1 publié par la division santé. Les réactions des pays au pré-rapport témoignent de la structuration du comité en deux groupes antagonistes : l'un plutôt favorable aux positions du BIAC, et intégrant la politique du médicament dans les politiques industrielles nationales, l'autre envisageant prioritairement le médicament comme un bien de santé publique. Dans le premier camp, le Japon et les États-Unis rappellent le «nécessaire équilibre entre la protection des brevets et l'accès aux médicaments », le délégué japonais ajoutant que, « comme l'a dit la déclaration ministérielle, il faut évidemment protéger les brevets si on veut créer de nouveaux médicaments ». La déclaration du délégué étasunien semble particulièrement attendue. S’il commence, de façon routinière, par féliciter l'OCDE pour ce travail, il rappelle toutefois la nécessité d'ouvrir les discussions «à toutes les parties prenantes », soulignant ce faisant que les industriels n'ont pas été assez consultés. Surtout, il achève son commentaire sur un ton menaçant :
«Les États-Unis financent beaucoup la recherche de base, notre pays paye très cher les produits pharmaceutiques. On a dit qu'il fallait tout mettre sur la table ; il faut plusieurs options politiques étant donné la diversité des systèmes nationaux. Je pense que l'OCDE aurait intérêt à prolonger les options politiques où il $\mathrm{y}$ a consensus. C'est un domaine très polémique où il est possible d'être extrême, ce qui ne peut pas permettre d'avancer. Gouvernement, indus- triels, consommateurs y gagnent, et c'est là que l'OCDE doit avancer. L'OCDE peut montrer toutes les options politiques mais doit se concen- trer sur les options consensuelles (il martèle les derniers mots $)^{70}$. »

Du côté des pays défendant le médicament comme « bien public », le pré-rapport est considéré « satisfaisant ». À la suite du premier tour de parole, une option politique « consensuelle » se dégage, autour de la nécessité de la coopération internationale dans les discussions avec les industries pharmaceutiques. Le délégué autrichien, par exemple, encourage l'OCDE :
"Ne soyez pas effrayés des éventuelles épreuves et continuez dans cette direction; nous savons tous qu'il y a du lobbying. [...] Il n'y a pas seulement des market failures ${ }^{71}$ mais aussi des policy failures. Les pays, la Commission, etc., travaillent-ils assez en collaboration pour augmenter les standards? Nous devons colla- borer! Nous ne devons pas avoir de clauses de confidentialité car elles minent les possibilités de collaboration [...] autrement les contribuables payent deux fois, une fois quand ils payent pour la R\&D pharma, et (une fois) pour les

66. Entretien avec un.e membre du comité d'expert.e.s, février 2018.

67. Voir "Médicaments. Des prix de malades ", Public Eye, 12, 2018, p. 14 sq. 68. Plaquette de présentation de l'événement.
69. Titulaire d'un Ph.D en pathobiologie de l'Université de Columbia, spécialiste de la biologie du cancer de l'immunologie, Schmeissner entre dans l'administration des États-Unis d'Amérique en 2004, d'abord comme conseiller au département de l'agri- culture, puis à l'office de la science et des technologies de la Maison Blanche avant d'intégrer la HHS.

70. Réunion du comité santé, 26 juin 2017. 71. Les market failures sont un terme d'économie de la santé qui désigne le fait que le marché pur de la théorie économique s'applique mal au secteur de la santé du fait des asymétries d'informations entre acheteurs et payeurs, et de la place de l'État dans le paiement et les politiques publiques de santé. 
médicaments [...] nous ne sommes pas contre les industries pharmaceutiques. [...] Les ministères de l'Économie et des Finances nous disent laissez-nous tranquilles, nous avons de bonnes industries, qui font de bonnes marges... Il faut que ça cesse ! Il faut que ça cesse, et que nous ayons des conversations constructives avec eux. Et vous (l'OCDE) il faut que vous continuiez et que vous soyez rigoureux (bold) dans cette recherche. »

Le rapport final n'est publié qu'en novembre 2018 du fait des obstacles et des changements cosmétiques proposés par les États-Unis afin d'en retarder la parution. L'introduction témoigne des hésitations des rédacteurs-rices du rapport, en soulignant d'emblée que « compte tenu du contexte complexe, le rapport ne présente pas de recommandations politiques fermes $»^{72}$. Se bornant à rappeler que les pays restent maîtres de leurs politiques nationales sur le médicament, le rapport se limite à des recommandations « consensuelles » : augmenter la transparence dans l'évaluation des médicaments et les échanges d'information sur leurs prix, augmenter la compétition sur les marchés des médicaments. Enfin, il évacue immédiatement la licence d'office, qui ne fait guère que l'objet d'un encadré, tandis que le paiement à la valeur est qualifié d'option «intéressante dans la mesure où elle permet à l'industrie d'être récompensée pour les médicaments les plus efficaces, et assure que le développement de médicaments à faible valeur ne sera pas surcompensé $\rrbracket^{73}$.

Le rapport a ainsi pour objectif de « contribuer au dialogue constructif entre les parties prenantes », mais ne propose pas de recommandations politiques contraignantes. Il s'avère en ce sens un argument pour le statu quo favorable aux industriels du médicament. Parmi les 19 «policy options » examinées, le point de vue adopté apparaît favorable aux industriels puisque sont reprises certaines de leurs revendications les plus anciennes (diminuer le temps d'attente avant la mise sur le marché, développer de nouveaux mécanismes d'incitation à l'innovation), tandis que, parmi les revendications des associations de patientee $s$ ou du TUAC, seule l'injonction principalement rhétorique à la « transparence » est retenue, mais en se contentant d'appeler à la bonne volonté des pays.

\section{"On a eu l'impression qu'ils se dégonflaient »}

« Ça aurait été étonnant que l’OCDE renverse la table, il ne faut pas se faire d'illusions. [...] Nous on a eu l'impression qu'ils se dégonflaient après avoir publié des choses intéressantes ${ }^{74}$. »

La structuration de cette "écriture collusive ${ }^{75}$ a mis aux prises, parfois brutalement, deux camps aux ressources asymétriques, représentés l'un par le BIAC et les ÉtatsUnis, l'autre par le TUAC et les ONG, pour la définition de la politique de prix du médicament. Si les arguments évoqués empruntent largement à la rhétorique de la science, les ressources scientifiques mobilisées, que ce soit par les délégations nationales, le BIAC, le TUAC, ou lors des consultations d'expert·e's extérieur·e's ne sont que de peu de poids face à la potentialité d'une défection, notamment étasunienne, qui n'a jamais à être évoquée - ou alors de façon extrêmement euphémisée - pour contraindre les rédacteurs rices à encastrer la politique de prix du médicament dans les politiques industrielles du plus gros bailleur de l'OCDE. Hommage que le vice rend à la vertu, l'ouverture formelle dans la consultation des parties prenantes laisse jouer à plein leurs inégalités de ressources tant matérielles que cognitives.

Cette série d'observations rappelle que la puissance matérielle contraint, notamment en milieu international, l'autonomie des Secrétariats. Condamnée à ménager ses organismes contributeurs pour subsister, l'institution se conforme, en adoptant des positions favorables à ses bailleurs les plus puissants, à une modération de façade qui légitime en retour ses recommandations. En dépit des velléités des rédacteurs-rices du rapport, il ne s'agit pas tant pour l'OCDE d'afficher une position que d'exprimer un point de conciliation entre des positions antagonistes, et inégalement aptes à se faire reconnaître comme vraies. C'est ainsi que le dépassement des oppositions dans des lieux communs inoffensifs, mais sans cesse décrits comme novateurs, apparaît à l'analyse comme la condition pour devenir - ou demeurer - un lieu neutre de l'expertise internationale. Mais si elle se donne pour juste et modérée, l'opposition rhétorique des positions extrêmes reconduit pourtant le statu quo, retardant d'autant la structuration internationale d'une politique coordonnée de régulation des industriels, au plus grand profit de ces derniers : l'absence de contraintes réglementaires perpétue, de fait, leur domination. 